\title{
The impact of obesity on cardiovascular disease risk factor
}

\author{
Arun Kumar \\ Professor and Head, Department of Biochemistry, Jagannath Gupta Institute of Medical Sciences, Buita, Budge Budge,
} Kolkata-700137

Obesity has emerged as the most potential cardiovascular risk factor and has raised concern among public and their health related issues not only in developed but also in developing countries. The Worldwide obesity occurrence has almost has gone three times since 1975. Research suggests there are about 775 million obese people in the World including adult, children, and adolescents. Nearly $50 \%$ of the children who are obese and overweight in Asia in are below 5 years. There is a steep incline of childhood obesity when compared to 1971 which is not only in developed countries but also in developing countries. A considerable amount of weight gain occurs during the transition phase from adolescence to young adulthood. It is also suggested that those adults who were obese in childhood also remained obese in their adulthood with a higher metabolic risk than those who became obese in their adulthood. In India, the urban Indian female in the age group of 30-45 years have emerged as an "at risk population" for cardiovascular diseases. To understand how obesity can influence cardiovascular function, it becomes immense important to understand the changes which can take place in adipose tissue due to obesity. There are two proposed concepts explaining the inflammatory status of macrophage. The predominant cause of insulin resistance is obesity. Epidemiological and research studies have indicated that the pathogenesis of obesity-related metabolic dysfunction involves the development of a systemic, low-grade inflammatory state. It is becoming clear that targeting the pro-inflammatory pathway may provide a novel therapeutic approach to prevent insulin resistance, particularly in obesityinduced insulin resistance. Some cost effective interventions that are feasible by all and can be implemented even in low-resource settings includes - population-wide and individual, which are recommended to be used in combination to reduce the greatest cardiovascular disease burden. The sixth target in the Global NCD action plan is to reduce the prevalence of hypertension by $25 \%$. Reducing the incidence of hypertension by implementing population-wide policies to reduce behavioral risk factors. Reducing cigarette smoking, body weight, blood pressure, blood cholesterol, and blood glucose all have a beneficial impact on major biological cardiovascular risk factors. A variety of lifestyle modifications have been shown, in clinical trials, to lower blood pressure, includes weight loss, physical activity, moderation of alcohol intake, increased fresh fruit and vegetables and reduced saturated fat in the diet, reduction of dietary sodium intake, and increased potassium intake. Also, trials of reduction of saturated fat and its partial replacement by unsaturated fats have improved dyslipidaemia and lowered risk of cardiovascular events. This initiative driven by the Ministry of Health and Family Welfare, State Governments, Indian Council of Medical Research and the World Health Organization are remarkable. The Government of India has adopted a national action plan for the prevention and control of non-communicable diseases (NCDs) with specific targets to be achieved by 2025 , including a $25 \%$ reduction in overall mortality from cardiovascular diseases, a $25 \%$ relative reduction in the prevalence of raised blood pressure and a $30 \%$ reduction in salt/sodium intake. In a nutshell increased BMI values can predict the nature of obesity and its aftermaths in terms inflammation and other disease associated with obesity. It's high time; we must realize it and keep an eye on health status in order to live long and healthy life.

Key words: Body mass index; Waist to hip ratio; Cardiovascular risk; Global Prevalence; Asian; Indian; Obesity; Obesity induced inflamation; Preventional Strategies

\section{Access this article online}

Website:

http://nepjol.info/index.php/AJMS

DOI: 10.3126/ajms.v10i1.21294

E-ISSN: 2091-0576

P-ISSN: 2467-9100 


\section{INTRODUCTION}

BMI is the body mass divided by the square of the body height, and is universally expressed in units of $\mathrm{kg} / \mathrm{m}^{2}$, resulting from mass in kilograms and height in metres. The BMI basically indicates the quantity of tissue mass viz, muscle, fat, and bone in a subject. Based on the BMI values, the subjects are categorized as underweight, normal weight, over-weight or obese. The widely accepted categories based on the values are underweight; $<18.5 \mathrm{~kg} / \mathrm{m}^{2}$, normal weight; $18.5-25 \mathrm{~kg} / \mathrm{m}^{2}$, overweight- 25 to $30 \mathrm{~kg} / \mathrm{m}^{2}$, obese- $>30 \mathrm{~kg} / \mathrm{m}^{2}$. These cut-off values does not necessarily indicates risks among all globally, as it is observed that Asians especially Indians have higher risks due to other compounding factors such as type 2 diabetes and cardiovascular disease as an additive risk at BMIs lower than the WHO cut-off point for overweight, $25 \mathrm{~kg} / \mathrm{m}^{2}$.

\section{Which is better indicator of risk: BMI or waist to hip ratio?}

As it is already established and widely accepted that increased blood pressure, higher ratio of total cholesterol to high-density lipoprotein are considered as standard markers of risk for cardiovascular diseases. Evidence shows that BMI; normal $\leq 25 \mathrm{~kg} / \mathrm{m}^{2}$ and waist-to-hip (W/H) ratio measurements (normal: women $\leq 0.8$, men $\leq 0.9$ ) may serve as a better atherogenic prognosticators. Studies conducted study among obese women, $\mathrm{W} / \mathrm{H}$ ratio was considered as better indicator of peer cardiac status than BMI. ${ }^{1}$ Waist to hip ratio was not only significantly connected to blood pressure and total cholesterol/HDL-cholesterol but also to triacylglycerols where as BMI was significantly associated to total cholesterol/HDL-cholesterol which is indicative of better foreteller than depending on mere BMI values. ${ }^{2}$

In a case-control study conducted among a total of 26903 participants who presented with their first myocardial infarction (MI) within 24 hours of symptoms onset from 52 countries $^{3}$ who were age/sex matched and with no history of CVD. The researchers observed that though BMI values were directly related to the risk of $\mathrm{MI}$, but the relation disappeared after adjustment of $\mathrm{W} / \mathrm{H}$ ratio and other risk factors.

\section{Obesity as a risk factor for cardiovascular diseases} The term "risk factor" includes modifiable life styles and biochemical and physiological characteristics as well as non modifiable personal characteristics such as age, gender and family history of early onset of cardiovascular disease (CVD) found in healthy individuals, which are independently related to the subsequent occurrence of coronary heart Disease (CHD) (Figure 1). The effects of risk factors in adults are additive i.e. the greater the number of high risk factors present, the greater the risk of CVD.

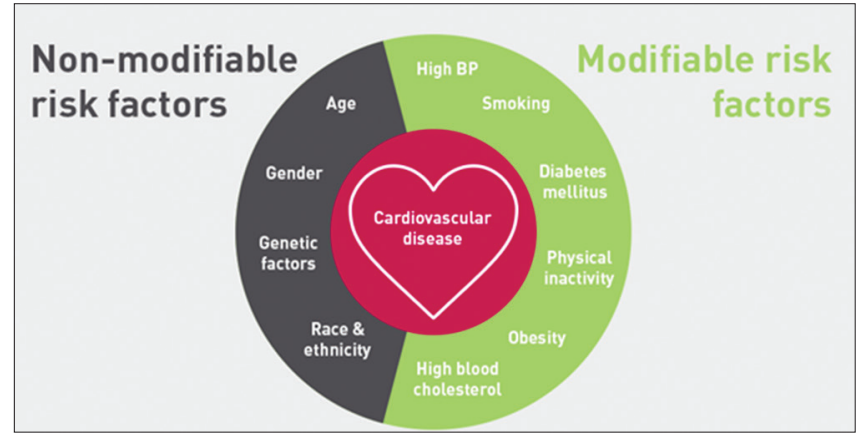

Figure 1. Risk factors of cardiovascular disease

Obesity has emerged as the most potential cardiovascular risk factor and has raised concern among public and their health related issues not only in developed but also in developing countries. ${ }^{4}$ As per reports by World Health Organization, more than 1400 million adults across the globe were overweight in 2008, and obesity being the most prevalent among more than 200 million men and 300 million women world-wide. ${ }^{5}$ Two-third of the world's population lives in countries where the most significant cause of death is due to obesity related illness. ${ }^{6}$ Being overweight in adulthood increases the risk of cardiovascular disease.

\section{Obesity-global prevalence}

The Worldwide obesity occurrence has almost has gone three times since 1975 . In 2016 $>1.9$ billion adults $>18$ years were overweight. ${ }^{7}$ Among them $>650$ million were obese. Thirty-nine percent (39\% of men and $40 \%$ of women) of adults aged $\geq 18$ years were overweight in 2016 , and overall about $13 \%$ of the world's adult population (11\% men and $15 \%$ of women) were obese. ${ }^{8}$ Most of the world's population lives in countries where overweight and obesity (Figure 2)are silent killers rather than underweight. ${ }^{9}$ Forty-one million children $<5$ years were overweight or obese in 2016. Over 340 million children and adolescents aged 5-19 were overweight or obese in 2016. Once was once considered as the disease of the developed country, but now it is also predominant in lower and middle income countries, more in urban localization. ${ }^{7}$

\section{Obesity among other countries}

New research suggests there are about 775 million obese people in the World including adult, children, and adolescents. ${ }^{10}$ Research findings suggest that there are nearly 650 million obese adults on the planet and about 125 million obese children and adolescents in the entire world according to a BMI values of $>30 .{ }^{11}$ The most obese countries are USA, China and India ranks third among the list. More than 50 percent of the world's 775 million obese people live in just ten of these countries: United States, China, India, Russia, Brazil, Mexico, Egypt, Germany, 


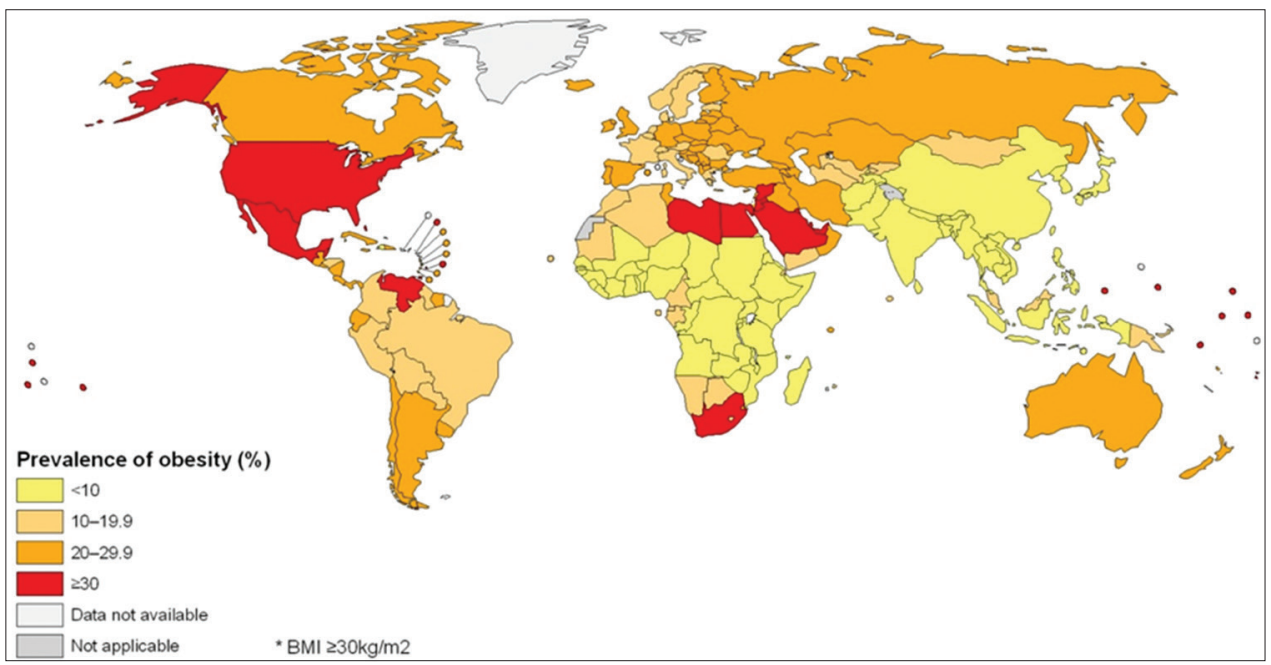

Figure 2. Global prevalence of obesity

Pakistan and Indonesia. ${ }^{11}$ The highest proportion of the world's obese people, 13 percent, live in the United States. ${ }^{6}$ In Africa, there is almost $50 \%$ increased overweight children since 2000 . Nearly half of the children who were obese and overweight in Asia in 2016 were below 5 years.

Obesity a major health threat among indians

In India, the urban Indian female in the age group of 30-45 years have emerged as an"at risk population" for cardiovascular diseases. ${ }^{12}$ Ninety-five percent of them are overweight or obese. Cardiovascular diseases in women are slowly on the rise and now considered as epidemic. Heart ailments have replaced communicable diseases in both rural and urban India. Indian urban women are at more risk today than they were three years ago. The reasons for the high cardiovascular diseases in women includes, an unhealthy lifestyle, increased intake of food containing high trans fat, cheese, sugar and salt. ${ }^{13}$ Inadequate physical exercise and stressful life, dependence onalcohol and tobacco, untimely dinners are the leading cause of increased abdominal obesity and increased $\mathrm{W} / \mathrm{H}$ ratio which is a major risk factor for heart diseases. ${ }^{14}$

The largest groups of women at risk of cardiovascular diseases are aged between 35 to 44 years. ${ }^{15}$ Low HDL-c and high BMI are the most common harbingers of CVD.

Cardiovascular risk is as high amongst housewives as it is amongst working professionals. The symptoms exhibited by women are also different than those by men. Women are less likely than men to have typical angina. They have higher false positive rate in treadmill test. Heart disease in women generally presents 10 years later than men but with greater risk than men. These premature deaths from CVD could be avoided if tobacco use, unhealthy diet, physical inactivity and harmful use of alcohol are controlled.
Obesity among children in other countries

There is a steep incline of childhood obesity when compared to 1971 which is not only in developed countries but also in developing countries. ${ }^{16}$ The occurrence of childhood obesity among Mediterranean countries surpasses the Scandinavian countries; nonetheless, the proportion of obese children is rising in both regions. Childhood obesity observed the Middle East, Central and Eastern Europe has the higher prevalence and Iran tops the list of childhood prevalence countries. ${ }^{17}$ Among developed countries, North America, Great Britain and some South Western European Countries (Greece, Italy, Malta, Portugal, Spain) the prevalence of overweight youth (10-16 years) was > 15\%. In Egypt and Cyprus, 14\% and $25 \%$ of $6-11$ year old children were reported to be overweight or obese. Obesity is increasingly being reported from countries such as Taiwan and Saudi Arabia. In Saudi Arabia, one in every six children aged 6 to 18 years old is obese. ${ }^{18}$ The incidence of obesity at all ages is on the rise in the developing countries including India. Reports from South India validates $21.4 \%$ of boys and $18.5 \%$ of girls aged between 12-14 years were overweight or obese. ${ }^{19}$ The prevalence of obesity among School children in India has been reported between $5.74 \%$ and $8.82 \% .{ }^{17}$ During last two decades, the prevalence of overweight among children and adolescents havealmost doubled in USA and tripled in Canada and Brazil. ${ }^{20} \mathrm{It}$ is observed from studies that those overweight adolescent have a strong tendency to become overweight in their adulthood and follow up studies observed that these adolescent are more likely to be affected by cardiovascular mortality. ${ }^{20}$

\section{Epidemiology and global scenario of obesity}

Obesity has reached epidemic proportions in the developed countries harboring around $30-40 \%$ of obese adults and children. ${ }^{21}$ World health organization has declared obesity 
as one of the most neglected diseases. The 2002 World Health Report lists overweight as the fifth most serious risk factor for both developed countries and developing countries. According to the report of International Obesity Task Force (IOTF), in the year 2000 about 10\% (a total of 155 million) of the young people aged 5-17 years globally were overweight; among whom 2-3\% (30-45 million) were obese, a further 22 million younger children are also affected according to previous IOTF global estimates based on WHO data for under fives. ${ }^{20} \mathrm{It}$ is predicted that the levels of obesity will continue to rise unless action is taken now. WHO has warned "the growth in the number of severely overweight adults to double that of underweight during 1995-2025" (WHO 1998). ${ }^{17}$ From existing data it has been projected that by the year 2030 levels of obesity could be as high as $50-80 \%$ in the USA, between 30-40\% in Australia, England and Mauritius and over $20 \%$ in some developing countries.

\section{Obesity among children}

Over the past 30 year period, the world wide prevalence of obesity in childhood has drastically increased. Globally in 2010, around 43 million children $<5$ years were over-weight. In a study comprising of children aged 1-17 years, overweightincreased the chances for prehypertension by $50 \%$ and doubledor tripled the figures in terms of hypertension, compared with normalweight children.Hypercholesterolemia in childhood is common in westernized countries with high rates of CHD. Atherosclerosis embarks at the age of nine years, evidence and supported by the study of cross sectional area of common carotid artery wall and the mean intima thickness of internal carotid arteries, which shows progressive increase in thickness due to fat deposition from lean to obese children. Autopsy studies have shown that early coronary atherosclerosis often begin in childhood and adolescence. ${ }^{22}$ Further, hypercholesterolaemia in adolescents correlated positively with changes in vasculature predictive of later $\mathrm{CVD} .{ }^{23}$ There is a tendency to persistence in ranks (tracking) for serum total and B-lipoprotein cholesterol with age.

A considerable amount of weight gain occurs during the transition phase from adolescence to young adulthood. It is also suggested that those adults who were obese in childhood also remained obese in their adulthood with a higher metabolic risk than those who became obese in their adulthood. ${ }^{24}$ It has been found that maternal obesity is the most significant predictor of obesity during childhood. ${ }^{25}$ Thus the combination of having an obese mother and an earlier onset of obesity translates into higher BMI and weight at young adulthood. Risk parameters for cardiovascular disease in childhood such as BMI, cholesterol, blood pressure and triglyceride concentrations have shown to be significantly correlated with adult levels over long term follow-up.
Childhood obesity causes increase in fasting insulin concentration to almost 12 manifoldin obese children aged 5 to 17 years. ${ }^{26}$ In addition, amounts of triglycerides, totalcholesterol, low density lipoprotein cholesterol (LDLcholesterol), and high density lipoprotein-cholesterol (HDL-cholesterol) are all more likely to be abnormal in overweightchildren than in normal weight children. Therefore, childhood health could greatly affect the risk ofcardiovascular disease in adulthood.

\section{Causes of obesity among children}

The fundamental cause of obesity and overweight is an energy imbalance between calories consumed and calories expended. Studies have confirmed that there is a decline in daily physical education classes in high school students with some schools removing the period of physical education from their time table, down trends in the number of children walking or cycling to school, and increase in sedentary lifestyles such as use of computers and mobiles phone games which limits their physical activity and outdoor games.

\section{Obesity - major contributory factor for cardiovascular diseases}

As we gain additional fat in the body, it forms an additional very active metabolic organ and these organs constantly generate very harmful substances called as free radicals or the active oxidant species. ${ }^{27}$ Excessive formation of free radicals damages the cells and this process is called as oxidative stress. Oxidative stress than triggers an inflammatory process which can affect the cells of throughout the whole body as they circulate in the blood stream. When trying to protect from these harmful processes, the target cells become resistance to action of insulin, which is also known as insulin resistance. If we are constantly exposed to these free radicals in our modern life style, such as high fat meals, inadequate sleep, stressful job, lack of exercise, sedentary life style, smoking consequently leads to low-grade chronic inflammation can cause havoc in our body. ${ }^{27}$ These chain reaction of free radical formation, oxidative stress, insulin resistance and inflammation is an interactive destructive metabolic cycle whereby we develops most of the diseases of life style namely diabetes, hypertension, cardiovascular diseases and cancer.

An individual's genetic make-up is one of the most important probabilities of developing certain diseases, including cardiovascular disease. The foundations of adult health are predestined in early stages of their life, much even before birth. It is most likely that poor young mothers with low educational status are most likely to give birth to low-birth-weight baby and less likely to breastfeed. These low-birth-weights are usually associated with increased risk of developing coronary heart disease, stroke and high 
blood pressure. Healthy life styles, good health-related habits,exercise, are all learnt early in life by the nurture and care of sensible peer and parents. Socioeconomic group is another factor which plays a vital role in future risk of CVD. Earlier research conducted has shown that males between 20-64 years of age in semi- and unskilled manual occupations have 3 folds higher risk of pre-mature death due to CVD. Moreover, when improvements to health do occur, the benefits are unevenly distributed within society. Demanding white color jobs and stressful life is associated with CVD and depression being another predictor of poor life expectancy among those who suffer from cardiovascular diseases. High salt intake leading to hypertension and consumption of saturated fat and transfat furthermore increases the risk of CVD. Overweight and obese are especially predisposed to develop CVD. Physical activity of at least 150 minutes each week is estimated to decrease the CVD risks by 30\% further reducing the risk of stroke and hypertension. Tobacco use in any form is estimated to cause about $10 \%$ of cardiovascular disease worldwide. Alcoholconsumption also increases the risk of cardiovascular diseases. Diabetes is yet another major risk factor and trigger for cardiovascular disease. Due to urbanization the trend of people is towards unhealthy diets and diverting from the good habits which causes serious implications of obesity.

Mechanism of obesity induced cardiovascular diseases To understand how obesity can influence cardiovascular function, it becomes immense important to understand the changes which can take place in adipose tissue due to obesity. When we consume high calorie diet it causes expansion of adipose tissue due to increase in the number of adipocytes which is mediated by proliferation of adipogenic progenitors. ${ }^{28} \mathrm{This}$ increase in adipocytes can result in an increase in adipocyte size affecting the normal metabolic functions of adipose tissue. In case of advanced stage of obesity, these hypertrophied adipocytes may undergo some necrotic or apoptotic changes contributing to the generation of inflammatory cells, macrophages and cellular dysfunction of adipose tissue. ${ }^{29}$ Other cells surrounding the adipocytes namely lymphoctyes, macrophages, fibroblasts and vascular cells also plays an important role in manipulating the functional status of adipose tissue. ${ }^{30}$ The increased macrophages around the adipocytes lead to higher macrophage-to-adipocyte ratio. This can further cause systematic inflammation along with insulin resistance.

There are two proposed concepts explaining the inflammatory status of macrophage, namely M1/M2. (Figure 3) Macrophages that accumulate in adipose tissue due to inflammation process in obese organisms usually expresses genes associated with M1-like or classically

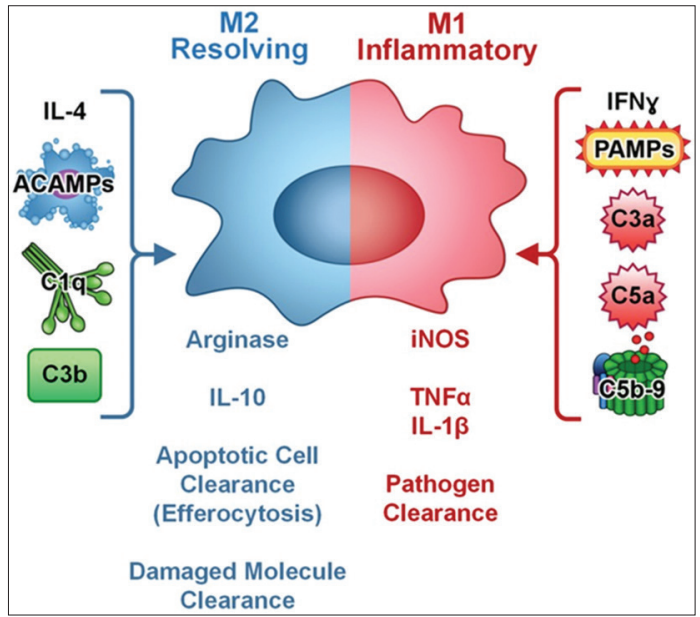

Figure 3. Complement regulating macrophage in inflammatory process

activated phenotype. On the other hand, macrophages accumulated in adipose tissue of lean organism tend to express genes associated with M2-like or alternatively activated phenotype. ${ }^{31}$

M1-like macrophages are usually associated with inflammation and tissue destruction due to production of tumor necrosis factor (TNF) $\alpha$, it also expresses inducible nitric oxide synthase (iNOS) and produce high levels of reactive oxygen and nitrogen intermediates. ${ }^{32} \mathrm{On}$ the contrary, M2-like macrophages are basically inclined towards wound healing, angiogenesis and resolution of inflammation due to expression of anti-inflammatory cytokines, namely interleukin (IL)-10, and the enzyme arginase-1 which inhibits iNOS activity. ${ }^{33} \mathrm{It}$ is thought that M1-like macrophages supports insulin resistance, whereas M2-like macrophages protect against obesity-induced insulin resistance. ${ }^{34}$

Another distinguishing feature of adipose tissue from obese organism is the presence of crown-like structures in histological sections..$^{35}$ Thesecrowns like structures represent macrophages around dead or dying adipocytes. Subjects lacking these crowns like structures in histological sections have better metabolic control, diminished expression of inflammatory gene and reduced cardiovascular risk than those who display such in histological sections.

The $\mathrm{T}$ cells in adipose tissue regulate the phenotypic feature of macrophage which is influenced by the grade of obesity in individuals. ${ }^{36} \mathrm{CD} 4+$ regulatory $\mathrm{T}$ cells and TH 2-polarized cells which are predominant in the adipose tissue of lean mice, regulates the adipose tissue functions and insulin sensitivity. ${ }^{37} \mathrm{On}$ the other hand, in obese state, the accumulation of CD8+ effector $\mathrm{T}$ cells and CD4+ TH1 cells in the adipose tissue generates TH1 signals which initiate the recruitment and activation of 
macrophages, perpetuate the pro-inflammatory cascade that is associated with insulin resistance. ${ }^{38}$ Thus, what is observed that the balance of TH1- and TH2-type signalare mainly influenced by obesity, which directly or indirectly influence macrophage recruitment and phenotype in adipose tissue, thereby generating either a pathogenic or a protective environment. Similarly, B cells also play a pivotal role in obesity-induced adipose tissue inflammation, promoting the $\mathrm{T}$ cell and macrophage activation and thus contributing to insulin resistance. ${ }^{39} \mathrm{Neutrophil}$ infiltration and activation also contributes to adipose tissue dysfunction through the action of neutrophil elastase. Research studies reported the role of eosinophils in adipose tissue to promote alternative activation of macrophages and glucose tolerance through the production of the TH2 cytokine IL-4. ${ }^{40}$ Therefore, the status of immune cells in adipose tissue is an area of thrust for research which needs to be further validated and investigated for better understanding of the complex relationship between inflammation and metabolism.

The phenotypic changes in adipose tissue due to obesity are presumed to induce vascular endothelial cell activation and recruitment of inflammatory cells. It can be justified that obesity promotes a vicious cycle of endothelial activation and tissue inflammation that contributes to adipose tissue dysfunction. Supporting this fact, studies shows that endothelial cells expresses cellular adhesion molecules such as P-selectin, E-selectin, and intercellular adhesion molecule 1 (ICAM-1). ${ }^{41}$ Administration of anti-ICAM-1 antibody can be used as a tool to prevent leukocyteendothelium interactions and macrophage infiltration into adipose tissue. In addition, adipose tissue inflammation also causes endothelial cell activation through inflammatory adipokines such as $\mathrm{TNF} \alpha$, which could contribute to obesity-associated cardiovascular disease.

\section{Obesity induced Insulin resistance}

The pancreas is a glandular organ of the digestive system and has dual functions, both exocrine and endocrine. Classically they have endocrine role related to blood glucose maintenance via secretion of insulin and other hormone and an exocrine role related to secretion of digestive substances involved in digestion process. Insulin is an anabolic hormone that promotes glucose uptake via Insulin dependent receptor, glycogenesis, lipogenesis and protein synthesis of skeletal muscles and fat tissue through the tyrosine kinase receptor pathway. Insulin maintains the blood glucose homeostasis and counteracts glucagon, epinephrine, glucocorticoids and growth hormone. So when insulin resistance occurs due to obesity and inflammation, it disrupts all insulin-mediated signaling pathways resulting in systemic hyperglycemia along with other metabolic symptoms like hypertension and hyperlipidemia.
To understand insulin resistance, we need to clarify molecular mechanisms of insulin signaling. At the molecular level, insulin binds to the cell surface insulin receptor that exists as a $\alpha 2 \beta 2$ heterodimer. Following the binding of insulin the tyrosine kinase domain of $\beta$ subunits autophosphorylates and activates its intrinsic kinase activity to proximal substrates such as insulin receptor substrate (IRS) ${ }^{42}$ family (IRS1-IRS4), Insulin sensitivity and secretion are reciprocally regulated, thus in case of insulin resistance we observe there is a increased insulin secretion to maintain normal blood glucose and lipid homeostasis. Several mediators are thought to be involved in passing a signal to the pancreatic beta cells to respond to insulin resistance, failure of those signals or inadequate adaptability results in inappropriate insulin levels resulting to complications of insulin resistance.

The potential signal mediators are glucose, free fatty acids, fat derived hormones (adiponectin) and gut hormone glucagon like peptide-1 (GLP-1). GLP-1 is an incretin hormone that is supposed to stimulate insulin secretion, causes beta cell mitosis while inhibiting apoptosis, inhibits glucagon secretion, delay gastric emptying and overall antidiabetic affects. ${ }^{43}$

The predominant cause of insulin resistance is obesity. It is associated with decreased number of receptors and postreceptor failure to activate tyrosine kinase. Due to insulin resistance there is an associated risk of cardiovascular disease and endothelial dysfunction.

\section{The adipokine concept}

Adipokines are a family of hormones and cytokines with both pro- and anti-inflammatory effect that are secreted by adipose tissue. These includes TNH- $\alpha$ and IL-6 (proinflammatory), leptin and adiponectin. Epidemiological and research studies have indicated that the pathogenesis of obesity-related metabolic dysfunction involves the development of a systemic, low-grade inflammatory state. ${ }^{44} \mathrm{An}$ excess of pro-inflammatory adipokines is hypothesized to explain the metabolic syndrome that accompanies obesity. Additionally several cell types with the myocardium express adipokines resulting in both autocrine and paracrine influences. Since TNF- $\alpha$ and IL- 6 inhibit adiponectin production, decreased circulating adiponectin is associated with left ventricular hypertrophy, suggesting that adiponectin might be cardioprotective. ${ }^{45}$ The degree of obesity could be presumed by the proportional increase in pro-inflammatory maker C-reactive protein and IL-6 which are predictive of development of type 2 diabetes. In contrast, weight loss leads to decreased levels of CRP and IL-6. These findings strongly suggest that obesity is highly associated with chronic low-grade inflammation, and it is believed that this obesity-linked inflammatory state is 
due to changes in the expression of cytokines by adipose tissue (Figure 4). ${ }^{46}$

Adipose tissue is mainly found in visceral and subcutaneous depots, it is also widely dispersed throughout the body. Other depot sites that might be a potential risk for cardiovascular disease include epicardial, perivascular, and pulmonary adipose tissue. The levels of adipokine production determine the adipose tissue depots it generally favor the production of pro-inflammatory adipokines regardless of depot site location. Interestingly, studies in mice suggest that aging irrespective of diet-induced obesity also induces the expression of proinflammatory adipokines, such as TNF $\alpha$ and IL-6 in visceral adipose tissue. ${ }^{47}$

Most adipokines which are identified till date are proinflammatory and they are upregulated in the obese state. Under conditions of obesity, these adipokines function to promote metabolic and cardiovascular diseases. Proinflammatory adipokines include TNF $\alpha$ leptin, IL-6, resistin, RBP4, lipocalin 2, IL-18, ANGPTL2, and others.

In this regard, it is very important to testify whether the increase in cardiovascular disease associated with an adipokine imbalance is due to a paracrine mechanism via local release of pro-inflammatory factors from epicardial or perivascular adipose tissue, or an endocrine mechanism that is reflected by an increase in serum adipokine levels.

Pro-inflammatory adipokine: TNF $\alpha$, leptin, interleukin-6 Adipose tissue is not only an energy storage organ but also an active endocrine tissue producing various biologically active proteins known as adipokines. Adipokines contributes to pathogenesis of obesity linked metabolic syndrome and cardiovascular complications. Omentin, a novel adipokine is a protein expressed and secreted from visceral but not subcutaneous adipose tissue that increases insulin sensitivity in adipocytes. Visceral and subcutaneous adipose tissue part from white adipose tissue, synthesizes unique adipokines which are mediators of inflammation

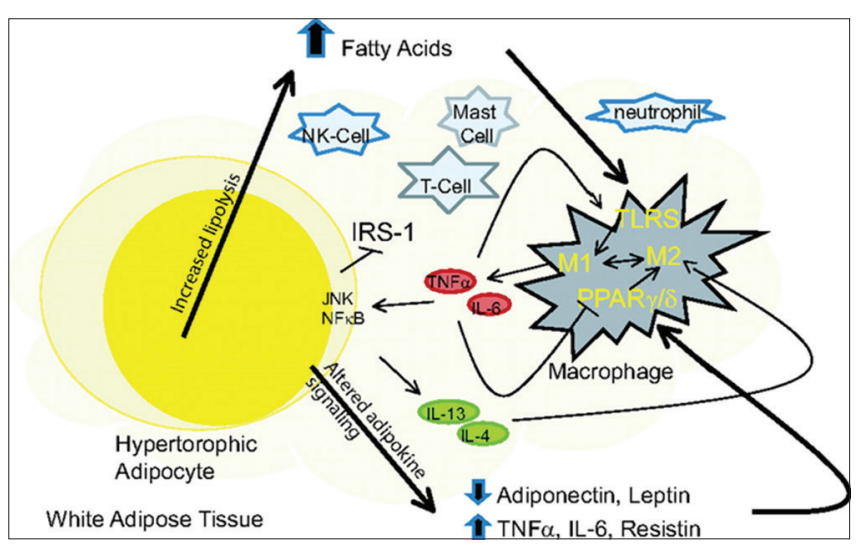

Figure 4. Signaling in inflammatory process due to obesity and insulin resistance in obese human and rodents. ${ }^{48}$ Research on this line was sparked by the discovery of adipsin, TNF- $\alpha$, andleptin which are bonafide adipokines.

TNF- $\alpha$ is key mediator and regulator of mammalian immune response in healthy organism and in diseased state. It was identified in the beginning as an endotoxininduced serum factor that mediates tumor necrosis and cancer cachexia. ${ }^{49} \mathrm{TNF}-\alpha$ is mostly expressed in monocytes and macrophages which are converted to their active form by TNF- $\alpha$ converting enzyme. TNF- $\alpha$ is a typical pro-inflammatory cytokine found to be elevated in obese humans and rodents suggesting its contribution to insulin resistance. ${ }^{50}$ Experimental evidences using cell lines treatment with TNF- $\alpha$ induces insulin resistance, and neutralization of TNF- $\alpha$ in obese enhances insulin sensitivity thus proving its role in inducing insulin resistance. ${ }^{51} \mathrm{TNF}-\alpha$ enhances the phosphorylation of IRS on Ser-307 residues that causes suppression of insulininduced IRS1 tyrosine phosphorylation and activation of downstream targets. ${ }^{52}$ Although TNF- $\alpha$ levels in the circulation is positively correlated with insulin resistance in other species, its clinical effects of TNF- $\alpha$ in human is still controversial. Short-term administration drugs blocking TNF- $\alpha$ in obese T2D patients suppresses inflammation but when the dosage is increased it does not shows improved insulin sensitivity. In contrast, long-term treatment of TNF- $\alpha$ blocking reagent in obese patients with severe inflammatory diseases improves insulin sensitivity. ${ }^{53} \mathrm{TNF}-\alpha$ also suppresses the expression of phosphodiesterase 3B (PDE3B) and perilipin. As PDE3B reduces cAMP after insulin stimulation, and perilipin regulates the access of hormone-sensitive lipase in adipocytes, TNF- $\alpha$ induces lipolysis in adipocytes to release free fatty acid. Free fatty acid in turn binds to TLR4, and pro-inflammatory factors are expressed through NF-KB activation..$^{53}$

\section{Leptin}

This is derived from the Greek meaning thin, the hormone which is responsible for energy expenditure. This is predominantly synthesized by adipose cells that balances energy by inhibiting hunger which are regulated by the hormone ghrelin also known as hunger hormone. Both hormones act on receptors present in the arcuate nucleus of the hypothalamus to regulate appetite in order to achieve energy homeostasis. In obesity, there is a decreased sensitivity to leptin, resulting in an inability to detect satiety despite high energy stores and high levels of leptin. ${ }^{54}$ Although the fat storage is the primary function of leptin, it plays a role in other physiological processes. The $\mathrm{Ob}(\mathrm{Lep})$ gene $(\mathrm{Ob}$ for obese, Lep for leptin) is located on chromosome 7 in humans. Any mutation in Leptin gene can cause extreme obesity in infancy with hyperphagia. Leptin is produced primarily in the adipocytes of white adipose tissue and 
also by brown adipose tissue, placenta, ovaries, skeletal muscle, stomach, mammary epithelial cells, bone marrow, gastric chief cells. The levels of leptin are influenced by the inflammatory markers and are involved in regulation of inflammatory response specifically to adipose-derived inflammatory cytokines. ${ }^{55}$ Chronically elevated leptin levels are associated with obesity, overeating, and inflammationrelated diseases, including hypertension, metabolic syndrome, and cardiovascular disease. ${ }^{56}$ Mutation of Obese gene increases food intake and obesity resulting in T2D. Importantly, exogenous administration of leptin to Lepob/ Lepob mice reduces obesity and restores insulin sensitivity. ${ }^{57}$ However, in circulation when leptin levels are increased in obese rodents and humans it suggests of leptin resistance. Leptin resistance has been reported as mediated by impaired leptin transport in blood brain barrier. ${ }^{58}$ Obesity-induced chronic inflammation also induces leptin resistance through the activation of TLR4, JNK, and IKK $\beta .{ }^{59}$ The structure of leptin is similar to pro-inflammatory helical cytokines including IL-2, IL-6, and granulocyte-colony stimulating factor (G-CSF), and leptin induces inflammatory responses through leptin receptor $\mathrm{b}$ (LepRb)and its proximal Janus kinase 2 (JAK2) and signal transducer and activator of transcription 3 (STAT3) signaling pathway. Leptin activates monocytes and macrophages to produce proinflammatory IL-6, TNF- $\alpha$, and IL-12 and stimulates the production of CCL2 and vascular endothelial growth factor in human hepatic stellate cells. TNF- $\alpha$ and LPSalso stimulate the expression of leptin and leptin receptor. Leptin also enhances the production of pro-inflammatory Th1 cytokines whereas suppresses the production of antiinflammatory Th2 cytokines such as IL-4 in CD4+ T cells. ${ }^{60}$

\section{Interleukin-6}

Interleukin-6 is a cytokine which is not only involved in inflammation and infection responses but also in the regulation of metabolic, regenerative, and neural processes. In classicsignaling interleukin- 6 stimulates target cells via a membrane bound interleukin- 6 receptor, which upon ligand binding associates with the signaling receptor protein gp130. Gp130 dimerizes, leading to the activation of Janus kinases and subsequent phosphorylation of tyrosine residues within the cytoplasmic portion of gp130.The cytokine interleukin-6 (IL-6) exhibits both pro- and antiinflammatory properties. Only few cells express the IL-6 receptor and respond to IL-6 (classic signaling), all cells can be stimulated via a soluble IL-6 receptor since gp130 is ubiquitously expressed. They are elevated in most, if not all, inflammatory states and have been recognized as potential targets of therapeutic intervention. The role of IL-6 in obesity and insulin resistance still remains controversial. IL-6 is expressed in adipose tissue and is well correlated with degree of obesity in humans. ${ }^{61}$ Peripheral administration of IL-6 interrupts insulin signaling due to enhance expression of SOCS3 in hepatocytes suggesting that obesity-induced IL-6 expression mediates insulin resistance..$^{53}$ On the contrary, IL-6 deficient mice demonstrate mature-onset obesity and hepatic inflammation, and IL- 6 administration reverses insulin resistance. ${ }^{62} \mathrm{As}$ central administration of IL-6 enhances energy expenditure and decreases obesity, IL-6 can also influence obesity and insulin sensitivity through a central nervous system mechanism. Thus the role IL-6 in obesity and insulin resistance likely depends upon the specific sites of expression that is integrated with other adipokine/cytokine factors in a systems integrated manner.

\section{Adiponectin: The anti-inflammatory adipokine}

Adiponectin are expressed by adipocytes exhibiting potent anti-inflammatory properties. Due to the overwhelming expressions of pro-inflammatory factors namely TNF- $\alpha$, IL-6, ROS, the expression of adiponectin in adipocytes are suppressed and decreased in obese rodents and humans. ${ }^{63}$ In contrast PPAR $\gamma$ antagonists stimulate the expression of adiponectin in adipocytes. Adiponectin activates AMP-dependent protein kinase (AMPK) through its receptors, ADIPOR1/2, to accelerate fatty acid oxidation and glucose uptake in muscle and to suppress gluconeogenesis in liver. ${ }^{64}$ Experiment evidence by administering exogenous adiponectin in transgenic mice results in improved insulin sensitivity whereas adiponectin deficient mice develop high fat diet induced inflammation and insulin resistance. ${ }^{65}$ Adiponectin inhibits LPS-induced TNF- $\alpha$ production in macrophages through inhibition of $\mathrm{NF}-x \mathrm{~B}$ activation and stimulate the production of anti-inflammatory IL-10. ${ }^{66}$ Adiponectin also promotes the differentiation of anti-inflammatory M2 macrophages and modulates $\mathrm{T}$ cells activation and inflammatory function of NK cells ${ }^{67}$ Adiponectin receptors are upregulated on the surface of human $T$ cells after stimulation by antigen and it mediates apoptosis of antigen specific $\mathrm{T}$ cells resulting in the suppression of antigen specific $\mathrm{T}$ cells expansion. Furthermore adiponectin suppresses TLR-mediated IFN- $\gamma$ production in NK cells without affecting in cytotoxicity of NK cells. ${ }^{68}$ Thus adiponectin can also delays and suppress the development of atherosclerosis, fatty liver diseases, and liver fibrosis. Low serum levels of adiponectin have been associated with coronary artery disease, hypertension, left ventricular hypertrophy, and a greater risk of myocardial infarction. ${ }^{69}$ Numerous experimental studies have shown that adiponectin exerts protective actions on cardiovascular cell types including vascular endothelial cells, smooth muscle cells, and cardiac myocytes, and adiponectindeficient mice display worse outcomes in various models of cardiovascular disease. ${ }^{70}$ Importantly, mouse models of adiponectin-deficiency display normal body and fat mass and normal metabolic parameters when fed a normal chow diet. Thus, the cardiovascular actions of adiponectin can be studied independently of its metabolic regulatory effects. 
As discussed, Obesity increases the inflammatory markers which causes insulin resistance in human, although observed that approximately $20 \%$ of the obese population remains fully insulin sensitive and metabolically normal regardless of inflammation. Though research evidence supportinflammation a prime cause of insulin resistance, still it is unclear what is the initiation factor(s) which is responsible for impulse of the inflammatory cascade. Research studies assumes that adipocyte released chemokines such as CCL2 is responsible for the initiation of proinflammatory macrophage infiltration. ${ }^{53}$ However, we now know that infiltration of macrophages is a late step in the adipose tissue inflammatory process and the earliest events is the infiltration of neutrophils. Whether or not neutrophil recruitment/activation in adipose tissue is the initiator of the inflammatory cascade and/or the signals responsible for neutrophil recruitment remain undetermined.

Though reports from enormous research have been published but still the queries remain unresolved in the mechanism/s by which adipose tissue inflammation results in liver and skeletal muscle insulin resistance. Several studies have also observed the local liver and skeletal muscle expression of pro-inflammatory cytokines and activation of inflammatory cells. Whether this results from systemic inflammation emanating from adipose tissue or is due to a local release of pro-inflammatory chemokines/cytokines has yet to be established. Moreover, whether this accounts for the hepatic and skeletal muscle insulin resistance or results from alterations in central signaling and/or systemic factors is area that needs further study.

It is becoming clear that targeting the pro-inflammatory pathway may provide a novel therapeutic approach to prevent insulin resistance, particularly in obesity-induced insulin resistance. In summary, adipose tissue has multiple integrative functions serving as energy storage organ that can provide fuel for energy production in times of external nutrient shortage. However, over the past two decades adipocytes have become established as bonafideprofessional endocrine cells that integrate whole body energy status with eating behavior, energy expenditure, and insulin sensitivity. Moreover, adipose tissue has become a central node for driving local and systemic sterile inflammation that is a key element in obesity-induced insulin resistance. Although many adipokines have been identified and well studied, the identification and functional studies of new adipokines and their control of integrative physiologic responses are essential to understand pathophysiological mechanisms of obesity-induced metabolic diseases.

Goals to decrease the premature deaths due to CVD Some cost effective interventions that are feasible by all and can be implemented even in low-resource settings includes - population-wide and individual, which are recommended to be used in combination to reduce the greatest cardiovascular disease burden.Examples of population-wide interventions that can be implemented to reduce CVDs include- Comprehensive tobacco control policies which can be implemented at the level of governance, mass campaigning and advertisements in television and radio regarding the effects of high fat diet in health, promoting cycling and walking to enhance physical activity, provide healthy meals in schools to children, funded by government, planning and documentation of guidelines to educate mass regarding the aftermath of obesity in various disease. At the individual level, for prevention of first heart attacks and strokes, individual health-care interventions need to be targeted to those at high total cardiovascular risk or those with single risk factor levels above traditional thresholds, such as hypertension and hypercholesterolemia. The former approach is more cost-effective than the latter and has the potential to substantially reduce cardiovascular events. This approach is feasible in primary care in low-resource settings, including by non-physician health workers.

For secondary prevention of cardiovascular disease in those with established disease, including diabetes, treatment with the following medications is necessary: aspirin, betablockers, angiotensin-converting enzyme inhibitors, statins.

The benefits of these interventions are largely independent, but when used together with smoking cessation, nearly $75 \%$ of recurrent vascular events may be prevented. Currently there are major gaps in the implementation of these interventions particularly at the primary health care level.In addition costly surgical operations are sometimes required to treat CVDs. They include: coronary artery bypass, balloon angioplasty, valve repair and replacement, heart transplantation, artificial heart operations. Medical devices are required to treat some CVDs. Such devices include pacemakers, prosthetic valves, and patches for closing holes in the heart.Under the leadership of the WHO, all Member States (194 countries) agreed in 2013 on global mechanisms to reduce the avoidable NCD burden including a "Global action plan for the prevention and control of NCDs 20132020 ". ${ }^{71}$ This plan aims to reduce the number of premature deaths from NCDs by $25 \%$ by 2025 through nine voluntary global targets. Two of the global targets directly focus on preventing and controlling CVDs.

Global action plan for the prevention and control of NCDs 2013-2020

The sixth target in the Global NCD action plan is to reduce the prevalence of hypertension by $25 \% \cdot{ }^{71}$ Raised blood pressure is one of the leading risk factor for cardiovascular disease. The global prevalence of hypertension in adults 
aged $\geq 18$ years is around $24.1 \%$ in men and $20.1 \%$ in women in $2015 .{ }^{72}$ The number of adults with raised blood pressure increased from 594 million in 1975 to 1.13 billion in 2015, with the increase largely in low- and middle-income countries. $^{73}$

Reducing the incidence of hypertension by implementing population-wide policies to reduce behavioral risk factors, including harmful use of alcohol, physical inactivity, overweight, obesity and high salt intake, is essential to attaining this target. A total-risk approach needs to be adopted for early detection and cost-effective management of hypertension in order to prevent heart attacks, strokes and other complications.

The eighth target in the Global NCD action plan states at least $50 \%$ of eligible people should receive drug therapy and counseling to prevent heart attacks and strokes. ${ }^{74}$ Prevention of heart attacks and strokes through a total cardiovascular risk approach is more cost-effective than treatment decisions based on individual risk factor thresholds only and should be part of the basic benefits package for pursuing universal health coverage. Achieving this target will require strengthening key health system components, including health-care financing to ensure access to basic health technologies and essential NCD medicines.

In 2015, countries set their national targets and measure progress on the 2010 baselines reported in the "Global status report on noncommunicable diseases 2014".

\section{Treatment and prevention}

There is little controversy over the benefits to cardiovascular health of not smoking, eating awell balanced diet, maintaining mental well-being, taking regular exercise and keeping active, asdemonstrated in large cohort studies. These health behaviors also play an etiological role in othernon-communicable diseases, such as cancer, respiratory disease, diabetes, osteoporosis and liverdisease, which makes interventions to promote them potentially very cost-effective. However, there is considerable uncertainty about the best ways of helping people at high CVD risk to modify their behavior.

Reducing cigarette smoking, body weight, blood pressure, blood cholesterol, and blood glucose all have a beneficial impact on major biological cardiovascular risk factors. Behaviors such as stopping smoking, taking regular physical activity and eating a healthy diet promote health andhave no known harmful effects. They also improve the sense of well-being and are usually lessexpensive to the health care system than drug treatments, which may also have adverse effects. A variety of lifestyle modifications have been shown, in clinical trials, to lower blood pressure. These include weight loss in the overweight, physical activity, moderationof alcohol intake, increased fresh fruit and vegetables and reduced saturated fat in thediet, reduction of dietary sodium intake, and increased potassium intake. Itis important to recognize, however, that most of the trials of lifestyle modification have been ofshort duration, which are unlikely to be feasible in routineprimary care in many countries. Still, the evidence supports the notion that it is possible to modify health behaviors and reduce blood pressure. More encouragingly, randomized trials, involving aprogram of weight reduction, dietary manipulation and physical activity, reduced the incidenceof type 2 diabetes among people at high risk of developing it. Also, trials of reductionof saturated fat and its partial replacement by unsaturated fats have improved dyslipidaemia and lowered risk of cardiovascular events.

Appropriatepolicies might address: agricultural subsidies for fruits and vegetables; food pricing and availability;labeling of food; public transport; pedestrian- and cyclist-friendly road planning; schoolhealth education; and tobacco control measures, including prohibition of advertising and pricecontrol. The overall objective should be to make it easy for the population to make healthy choicesrelated to diet, physical activity and avoidance of tobacco.

Strategies adopted by Indian government against CVD This initiative driven by the Ministry of Health and Family Welfare, State Governments, Indian Council of Medical Research and the World Health Organization. The Government of India has adopted a national action plan for the prevention and control of non-communicable diseases (NCDs) with specific targets to be achieved by 2025 , including a $25 \%$ reduction in overall mortality from cardiovascular diseases, a $25 \%$ relative reduction in the prevalence of raised blood pressure and a $30 \%$ reduction in salt/sodium intake. The funds will support costs associated with staffing, implementing and maintaining the India Hypertension Management Initiative. This structure helps patients receive the full benefit of the initiative, by ensuring the resources needed to control blood pressure are in place. Most of the costs are borne by state governments themselves, which pay the primary care staff, procure drugs and blood pressure monitors, and have overall responsibility for the program. The additional inputs will demonstrate their benefit, and that state governments may in the coming years decide to expand the program so that all patients in India can benefit from this life-saving treatment.

In a nutshell increased BMI values can predict the nature of obesity and its aftermaths in terms inflammation and other disease associated with obesity. It's high time; we must realize it and keep an eye on health status in order to live long and healthy life. 


\section{REFERENCES}

1. Elsayed EF, Tighiouart $H$, Weiner DE, Griffith J, Salem D, Levey AS, et al.Waist Hip Ratio and Body Mass Index as Risk Factors for Cardiovascular Events in Chronic Kidney Disease. Am J Kidney Dis. 2008 Jul; 52(1): 49-57.

2. Rudolf E Noble. Waist-to-hip ratio versus BMI as predictors of cardiac risk in obese adult women. West J Med 2001; 174(4): 240-241.

3. Andrew Mente, SalimYusuf, Shofiqul Islam, Matthew J. McQueen Supachai Tanomsup, Churchill L. Onen, Sumathy Rangarajan, HertzelC.Gerstein, Sonia S. Anand. Metabolic Syndrome and Risk of Acute Myocardial Infarction: A Case-Control Study of 26,903 Subjects From 52 Countries.Journal of the American College of Cardiology 2010; Volume 55, Issue 21: 2390-2398.

4. Ruth S.M Chan and Woo J. Prevention of Overweight and Obesity: How Effective is the Current Public Health Approach.Int J Environ Res Public Health 2010; 7(3): 765-783.

5. http://www.wpro.who.int/mediacentre/factsheets/obesity/en

6. http://www.healthdata.org/news-release/nearly-one-thirdworld\%E2\%80\%99s-population-obese-or-overweight-newdata-show

7. http://www.who.int/mediacentre/factsheets/fs311/en

8. Hruby A and Hu FB. The Epidemiology of Obesity: A Big Picture. Pharmacoeconomics 2015; 33(7): 673-689.

9. NCD Risk Factor Collaboration. Worldwide trends in body-mass index, underweight, overweight, and obesity from 1975 to 2016 : a pooled analysis of 2416 population-based measurement studies in 128.9 million children, adolescents, and adults. Lancet 2017; 390(10113): 2627-2642.

10. https://www.newswire.com/news/report-world-obesity-rankingssuggest-over-775-million-people-are-obese-20019901

11. Wilborn C, Beckham J, Campbell B, Harvey T, Galbreath M, La Bounty P, et al. Obesity: Prevalence, Theories, Medical Consequences, Management, and Research Directions.J IntSoc Sports Nutr 2005; 2(2): 4-31.

12. http://www.thehindu.com/news/cities/Delhi/urban-women-atrisk-population-for-heart-diseases/article7704215.ece

13. Anand SS, Hawkes C, de Souza RJ, Mente A, Dehghan M, Nugent R, et al. Food Consumption and its impact on Cardiovascular Disease: Importance of Solutions focused on the globalized food system. A Report from the Workshop convened by the World Heart Federation.J Am Coll Cardio 2015; 66(14): 1590-1614.

14. https://www.health.harvard.edu/staying-healthy/abdominalobesity-and-your-health

15. http://www.business-standard.com/article/sponsored-content/studyreveals-that-3-in-5-women-in-india-are-at-high-risk-of-cardiovasculardisease-as-early-as-35-years-of-age-114092900800_1.html

16. https://www.ncbi.nlm.nih.gov/books/NBK83830

17. Ahmad Q, Ahmad CB and Ahmad SM. Childhood Obesity. Indian J EndocrinolMetab 2010; 14(1): 19-25.

18. Omer EOM, Al Shehri MA and AI Bakri UM. Nutritional Status of Public Elementary School Boys in Al-Baha City, Saudi Arabia. J Nutr Food Sci 2013; 3:179.

19. Heidari K, Minasian V, Marandi SM , Kelishadi R, Khalighinejad P and Davari $H$. Prevalence of Obesity and Overweight in 12-14-year-old Students in Isfahan-Iran.Int J Prev Med 2014; 5(Suppl 2): S120-S125.

20. Wang Yand Lim $\mathrm{H}$. The global childhood obesity epidemic and the association between socio-economic status and childhood obesity.Int Rev Psychiatry 2012; 24(3): 176-188.

21. $22^{\text {nd }}$ European Congress on Obesity (ECO2015), Prague,
Czech Republic, May 6-9, 2015: Abstracts.Obes Facts 2015; 8(Suppl 1): 1-272.

22. Young Mi Hong. Atherosclerotic Cardiovascular Disease Beginning in Childhood. Korean Circ J. 2010; 40(1): 1-9.

23. Daniels SR, Pratt CA and Hayman LL. Reduction of Risk for Cardiovascular Disease in Children and Adolescents. Circulation 2011; 124(15): 1673-1686.

24. Biro FM and Wien M. Childhood obesity and adult morbidities. Am J ClinNutr 2010; 91(5): 1499S-1505S.

25. Williams CB, Mackenzie KC and Gahagan S. The Effect of Maternal Obesity on the Offspring. ClinObstetGynecol 2014; 57(3): 508-515.

26. Raj M and Krishna Kumar R. Obesity in children \& adolescents. Indian J Med Res. 2010; 132(5): 598-607.

27. Lobo V, Patil A, Phatak A and Chandra N. Free radicals, antioxidants and functional foods: Impact on human health. Pharmacogn Rev 2010; 4(8): 118-126.

28. Jung Uand Choi MS. Obesity and Its Metabolic Complications: The Role of Adipokines and the Relationship between Obesity, Inflammation, Insulin Resistance, Dyslipidemia and Nonalcoholic Fatty Liver Disease.Int J MolSci 2014; 15(4): 6184-6223.

29. Fuster JJ, Ouchi N, Gokce N and Walsh K. Obesity-induced Changes in Adipose Tissue Microenvironment and Their Impact on Cardiovascular Disease.Circ Res 2016; 118(11): 1786-1807.

30. Bai $Y$ and Sun $O$. Macrophage recruitment in obese adipose tissue. Obes Rev 2015; 16(2): 127-136.

31. Coats BR, Schoenfelt KQ, Barbosa- Lorenzi VC, Peris E, Cui C, Hoffman A, et al. Metabolically activated adipose tissue macrophages perform detrimental and beneficial functions during diet-induced obesity. Cell Rep 2017; 20(13): 3149-3161.

32. Martinez FO and Gordon S. The M1 and M2 paradigm of macrophage activation: time for reassessment.F1000Prime Rep 2014; $6: 13$.

33. Cassini-Vieira P, Araújo FA, da Costa Dias FL, Russo RC, Andrade SP, Teixeira MM, et al. iNOS Activity Modulates Inflammation, Angiogenesis, and Tissue Fibrosis in PolyetherPolyurethane Synthetic Implants. Mediators of Inflammation Volume 2015 (2015), Article ID 138461, 9 pages.

34. Mario A. R. Lauterbach and Thomas Wunderlich F. Thomas Wunderlich.Macrophage function in obesity-induced inflammation and insulin resistance. Pflugers Arch 2017; 469(3): 385-396.

35. Sun S, Ji Y, Kersten S and Ling Qi. Mechanisms of Inflammatory Responses in Obese Adipose Tissue. Annu Rev Nutr 2012; 32: 261-286.

36. Boutens $L$ and Stienstra R. Adipose tissue macrophages: going off track during obesity.Diabetologia2016; 59: 879-894.

37. Jin Young Huh, Yoon Jeong Park, Mira Ham and Jae Bum Kim. Crosstalk between Adipocytes and Immune Cells in Adipose Tissue Inflammation and Metabolic Dysregulation in Obesity.Mol Cells2014; 37(5): 365-371.

38. Cho KW, Morris DL, DelProposto JL, Geletka L, Zamarron B, Martinez-Santibanez G, et al. An MHC Class II Dependent Activation Loop Between Adipose Tissue Macrophages and CD4+ T cells Controls Obesity-Induced Inflammation. Cell Rep 2014; 9(2): 605-617.

39. Winer DA, Winer S, Chng MHY, Shen L and Engleman EG. $B$ Lymphocytes in obesity related adipose tissue inflammation and insulin resistance.Cell Mol Life Sci 2014; 71(6): 1033-1043.

40. Yi Zhang, Peng Yang, Ran Cui, Manna Zhang, Hong Li, Chunhua Qian, et al. Eosinophils Reduce Chronic Inflammation in Adipose Tissue by Secreting Th2 Cytokines and Promoting M2 Macrophages Polarization.Int J Endocrinol 2015; 565760.

41. Lorenzon P, Vecile E, Nardon E, Ferrero E, Harlan JM, Tedesco 
F and Dobrina A. Endothelial Cell E- and P-Selectin and Vascular Cell Adhesion Molecule-1 Function as Signaling Receptors.J Cell Biol 1998; 142(5): 1381-1391.

42. Gammeltoft $S$ and Obberghen EV. Protein kinase activity of the insulin receptor.Biochem J 1986; 235(1): 1-11.

43. Lee YS and Jun HS. Anti-Inflammatory Effects of GLP-1-Based Therapies beyond Glucose Control.Mediators Inflamm 2016; 3094642.

44. Rosário Monteiro. Chronic Inflammation in Obesity and the Metabolic Syndrome.Mediators Inflamm 2010; 289645.

45. Ouchi N, Shibata R and Walsh K. Cardioprotection by Adiponectin. Trends Cardiovasc Med 2006; 16(5): 141-146.

46. Caër C, Rouault C, Roy TL, Poitou C, Aron-Wisnewsky J, Torcivia A, et al. Immune cell-derived cytokines contribute to obesity-related inflammation, fibrogenesis and metabolic deregulation in human adipose tissue. Sci Rep 2017; 7: 3000.

47. Nakamura K, José J. Fuster and Walsh K. Adipokines: A link between obesity and cardiovascular disease.J Cardiol 2014; 63(4): 250-259.

48. Makki K, Froguel P and Wolowczuk I. Adipose Tissue in ObesityRelated Inflammation and Insulin Resistance: Cells, Cytokines, and Chemokines.ISRN Inflamm 2013; 2013: 139239.

49. Carswell EA, Old LJ, Kassel RL, Green S, Fiore N and Williamson B. An endotoxin-induced serum factor that causes necrosis of tumors.ProcNatlAcadSci U S A 1975;72(9):3666-70.

50. Kang YE, Kim JM, Joung KH, Lee J, You BR, Jeong Choi M, et al. The Roles of Adipokines, Proinflammatory Cytokines, and Adipose Tissue Macrophages in Obesity-Associated Insulin Resistance in Modest Obesity and Early Metabolic Dysfunction. PLoS One 2016; 11(4): e0154003.

51. Boucher J, Kleinridders A and Ronald Kahn C. Insulin resistance associated to obesity: the link TNF-alpha. Arch PhysiolBiochem 2008;114(3):183-94.

52. Boucher J, Kleinridders A and Ronald Kahn C. Insulin Receptor Signaling in Normal and Insulin-Resistant States.Cold Spring HarbPerspectBiol 2014; 6(1): a009191.

53. Kwon $\mathrm{H}$ and Pessin JE. Adipokines Mediate Inflammation and Insulin Resistance.Front Endocrinol (Lausanne) 2013; 4: 71.

54. Carter S, Caron A, Richard D and Picard F. Role of leptin resistance in the development of obesity in older patients. ClinInterv Aging 2013; 8: 829-844.

55. likuni N, Lam QKL, Lu L, Matarese G and La Cava A. Leptin and Inflammation.Currlmmunol Rev 2008; 4(2): 70-79.

56. Paul Holvoet. Stress in Obesity and Associated Metabolic and Cardiovascular Disorders. Scientifica (Cairo). 2012; 205027.

57. D'souza AM, Johnson JD, Clee SM and Timothy J. Suppressing hyperinsulinemia prevents obesity but causes rapid onset of diabetes in leptin-deficient Lepob/ob mice.MolMetab 2016; 5(11): 1103-1112.

58. Zhou $Y$ and Rui L. Leptin signaling and leptin resistance.Front
Med. 2013 Jun; 7(2): 207-222.

59. McArdle MA, Finucane OM, Connaughton RM, McMorrow AM and Roche HM. Mechanisms of Obesity-Induced Inflammation and Insulin Resistance: Insights into the Emerging Role of Nutritional Strategies.Front Endocrinol (Lausanne) 2013; 4: 52.

60. Matarese G, Moschos $S$ and Mantzoros CS. Leptin in Immunology. J Immunol 2005; 174 (6): 3137-3142.

61. Jung $U$ and Choi MS. Obesity and Its Metabolic Complications: The Role of Adipokines and the Relationship between Obesity, Inflammation, Insulin Resistance, Dyslipidemia and Nonalcoholic Fatty Liver Disease.Int J MolSci 2014; 15(4): 6184-6223.

62. Matthews VB, Allen TL, Risis S, Chan MHS, Henstridge DC, Watson $\mathrm{N}$, et al. Interleukin-6-deficient mice develop hepatic inflammation and systemic insulin resistance. Diabetologia 2010; Volume 53, Issue 11, pp 2431-2441.

63. Martin LJ, Woo JG, Daniels SR, Goodman E and Dolan LM. The Relationships of Adiponectin with Insulin and Lipids Are Strengthened with Increasing Adiposity.J ClinEndocrinolMetab 2005; 90(7): 4255-4259.

64. Yamauchi T, Kamon J, Minokoshi Y, Ito Y, Waki H, Uchida S, et al. Adiponectin stimulates glucose utilization and fattyacid oxidation by activating AMP-activated protein kinase.Nat Med 2002; 8(11):1288-95.

65. Kadowaki T, Yamauchi T, Kubota N, Hara K, Ueki K and Tobe K. Adiponectin and adiponectin receptors in insulin resistance, diabetes, and the metabolic syndrome. J Clin Invest 2006; 116(7): 1784-1792.

66. Santos F, José L,Rosaa C, Duarte G, Marilia P, Ana S, et al. Both adiponectin and interleukin-10 inhibit LPS-induced activation of the NF-KB pathway in 3T3-L1 adipocytes.Cytokine 2012; Volume 57, Issue 1: 98-106.

67. Luo $Y$ and Liu M. Adiponectin: a versatile player of innate immunity. J Mol Cell Biol 2016; 8(2): 120-128.

68. Kim KY, Kim JK, Han SH, Lim JS, Kim KI, Cho DH, et al. Adiponectin is a negative regulator of NK cell cytotoxicity.J Immunol 2006;176(10):5958-64.

69. Lee Y, Kim B, Lim YH, Kim MK, Choi BY and Shin J. The Relationship between Adiponectin and Left Ventricular Mass Index Varies with the Risk of Left Ventricular Hypertrophy.PLoS One 2013; 8(7): e70246.

70. Nakamura K, José J. Fuster and Walsh K. Adipokines: A link between obesity and cardiovascular disease.J Cardiol 2014; 63(4): 250-259.

71. http://www.who.int/nmh/events/ncd_action_plan/en

72. http://onlinelibrary.wiley.com/doi/10.1111/jch.13238/pdf

73. NCD Risk Factor Collaboration (NCD-RisC). Worldwide trends in blood pressure from 1975 to 2015: a pooled analysis of 1479 population-based measurement studies with $19 \cdot 1$ million participants. Lancet 2017;389(10064):37-55.

74. http://www.who.int/nmh/ncd-tools/target8/en

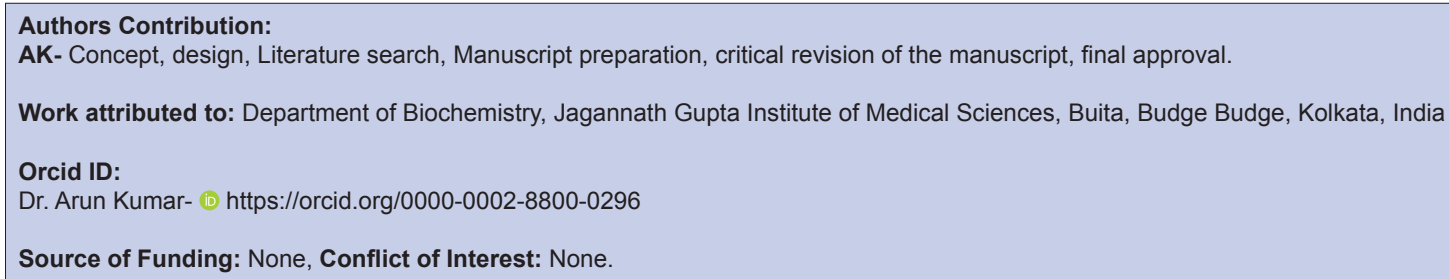

